\title{
LINKING CONFLICT TO INEQUALITY AND POLARIZATION ${ }^{1}$
}

\author{
By \\ Joan Esteban \\ Instituto de Análisis Económico (CSIC) \\ and \\ Debraj Ray \\ New York University \\ March 2009, revised March 2010
}

\begin{abstract}
In this paper we study a behavioral model of conflict that provides a basis for choosing certain indices of dispersion as indicators for conflict. We show that a suitable monotone transform of the equilibrium level of conflict can be proxied by a linear function of the Gini coefficient, the Herfindahl-Hirschman fractionalization index, and a specific measure of polarization due to Esteban and Ray.

JEL-Classification: D74, D31
\end{abstract}

Key-words: conflict, polarization, inequality.

\footnotetext{
${ }^{1}$ We are grateful to participants in the Yale Workshop on Conflict and Rationality, ESSET meeting at Gerzensee, CRED (Namur), GREMAQ (Marseille), IAE (Barcelona), and ISI (Delhi), and to Anja Sautmann for comments and research assistance. Joan Esteban is a member of the Barcelona GSE Research Network funded by the Government of Catalonia. He gratefully acknowledges financial support from the AXA Research Fund and from the Spanish Government CICYT project n. SEJ2006-00369. Debraj Ray is grateful for support from the Fulbright Foundation, and to the Indian Statistical Institute for warm hospitality during a sabbatical year.
} 


\section{INTRODUCTION}

In this paper we study a behavioral model of conflict that provides a basis for choosing certain indices of dispersion as indicators for conflict. We show that a suitable monotone transform of the equilibrium level of conflict can be proxied by a linear function of the Gini coefficient, the Herfindahl-Hirschman fractionalization index, and a specific measure of polarization due to Esteban and Ray.

Income inequality has been always viewed as closely related to conflict. In the Introduction of his celebrated book "On Income Inequality", Sen (1973) asserts that "the relation between inequality and rebellion is indeed a close one". Early empirical studies on the role of inequality in explaining civil conflict have focussed on the personal distribution of income or of landownership. ${ }^{2}$

Contemporary literature has shifted the emphasis from class to ethnic conflict. Here too the initial presumption has been that ethnic diversity is a key factor for ethnic conflict. Easterly and Levine (1997) used the index of fractionalization as a measure of diversity, and the measure has been used in several different empirical studies of conflict (see, e.g., Collier and Hoeffler, 2004, Fearon and Laitin, 2003, and Miguel, Satyanath and Sergenti, 2004). More recently, following on the idea that highly fragmented societies may not be highly conflictual, ${ }^{3}$ measures of polarization have also made their way into empirical studies of conflict. ${ }^{4}$

These contributions, while loosely based on theoretical arguments, are essentially empirically motivated in an attempt to identify a statistical regularity. The preference for

\footnotetext{
${ }^{2}$ See, for instance, Nagel (1974), Muller and Seligson (1987), Brockett (1992) or the survey article by Lichbach (1989).

${ }^{3}$ For instance, Horowitz (1985) argues that large cleavages are more germane to the study of conflict, stating that "a centrally focused system [with few groupings] possesses fewer cleavages than a dispersed system, but those it possesses run through the whole society and are of greater magnitude. When conflict occurs, the center has little latitude to placate some groups without antagonizing others."

${ }^{4}$ See Esteban and Ray (1994) and Wolfson (1994) for the earliest development of polarization measures, and Reynal-Querol (2002) for a special case of the Esteban-Ray measure which is then applied to a cross-section study of ethnic conflict by Montalvo and Reynal-Querol (2005a). See also the special issue of the Journal of Peace Research edited by Esteban and Schneider (2008), entirely devoted to the links between polarization and conflict.
} 
one particular index or another simply depends on its ability to fit the facts. In contrast, there is to our knowledge no behavioral model explaining why should we expect - to begin with - a relationship between the Gini or the fractionalization indices, and conflict. $^{5}$

In this paper we present a behavioral model of conflict that precisely defines the links between conflict and measures of dispersion, such as inequality and polarization. The model is general, in that it allows for conflict over both divisible private goods and (group-based) public goods. ${ }^{6}$ It is also general in that it allows for varying degrees of within-group cohesion, running the gamut from individualistic decisions (as in the voluntary contributions model) all the way to choices imposed by benevolent group leaders. Our main result is that a particular monotone transform of the equilibrium level conflict can be proxied by a weighted average of a particular inequality measure (the Gini coefficient), the fractionalization index used by Easterly and Levine and others, and a particular polarization measure from the class axiomatized by Esteban and Ray (1994). Moreover, the weights depend in a precise way on two parameters: the "degree of publicness" of the prize and the extent of intra-group "cohesion". In particular, our result suggests that if our derived equation were to be taken to the data, the estimated coefficients would be informative regarding these parameters.

While we link the severity of conflict to these measures, our paper does not address the issue of conflict onset. As discussed in Esteban and Ray (2008a), the knowledge of the costs of open conflict may act as a deterrent. For this reason we argue there that the relationship between conflict onset and the factors determining the intensity of conflict may not be monotonic. This issue is not contemplated here: we assume that society is in a state of conflict throughout.

We organize this paper as follows. Section 2 provides a very brief presentation of the basic measures of inequality and polarization. Section 3 develops a game-theoretic

\footnotetext{
${ }^{5}$ Esteban and Ray (1999) do discuss the possible links between polarization and equilibrium conflict in a model of strategic behavior. Montalvo and Reynal-Querol (2005b) also derive a measure of polarization from a rent-seeking game.

${ }^{6}$ The specific formulation is borrowed from Esteban and Ray (2001).
} 
model of conflict and some of its basic properties. The main result is obtained in Section 4 . Section 5 studies the accuracy of our approximation. Section 6 discusses the nature of the result and shows that it holds in a broader context. Section 7 concludes.

\section{INEQUALITY AND POLARIZATION}

Suppose that population is distributed over $m$ groups, with $n_{i}$ being the share of the population belonging to group $i$. Denote by $\delta_{i j}$ the "distance" between groups $i$ and $j$ (more on this below). Fix the location of any given group $i$ and compute the average distance to the other locations. The Gini index $G$ is the average of these distances as we take each location in the support as a reference point. ${ }^{7}$ We write it in unnormalized form $^{8}$ as

$$
G=\sum_{j=1}^{m} \sum_{i=1}^{m} n_{i} n_{j} \delta_{i j}
$$

We haven't been very specific about the distance $\delta_{i j}$. When groups are identified by their income, $\delta_{i j}$ could be the absolute or relative value of the income difference between $i$ and $j$. However, in principle we could apply this index to distributions over political, ethnic or religious groups. Unfortunately, in most cases where distance is non-monetary the available information does not permit a reasonable estimate of $\delta_{i j}$. This is why it is common to assume (sometimes implicitly) that $\delta_{i j}=1$ for all $i \neq j$ and, of course, $\delta_{i i}=0$. In that case, (1) reduces to

$$
F=\sum_{i=1}^{m} n_{i}\left(1-n_{i}\right)
$$

This is the widely used Hirschman-Herfindahl fractionalization index (Hirschman (1964)). It captures the probability that two randomly chosen individuals belong to different

\footnotetext{
${ }^{7}$ The properties of the Gini index are well known. Its first axiomatization is due to Thon (1982).

${ }^{8}$ The Gini is typically renormalized by mean distance; this makes no difference to the current exposition.
} 
groups. As mentioned before, this measure has been used to link ethnolinguistic diversity to conflict, public goods provision, or growth. ${ }^{9}$ At the same time, we know of no behavioral model of conflict that explicitly establishes a link between conflict and inequality (or fractionalization).

Esteban and Ray (1994) introduce the notion of polarization as an appropriate indicator for conflict. ${ }^{10}$ Their approach is founded on the postulate that group "identification" (proxied by group size) and intergroup distances can both be conflictual. Duclos, Esteban and Ray (2004) work with density functions over a space of characteristics to axiomatize a class of polarization measures, which we describe here for discrete distributions:

$$
P_{\beta}=\sum_{i=1}^{m} \sum_{j=1}^{m} n_{i}^{1+\beta} n_{j} \delta_{i j}, \text { for } \beta \in[0.25,1]
$$

An additional axiom, introduced and discussed by Esteban and Ray (1994), pins down the value of $\beta$ at 1 :

$$
P \equiv P_{1}=\sum_{i=1}^{m} \sum_{j=1}^{m} n_{i}^{2} n_{j} \delta_{i j}
$$

Because (4) is not derived formally for the model studied in Duclos, Esteban and Ray (2004), we provide a self-contained treatment in the Appendix.

The formal properties of this measure are discussed in detail in Esteban and Ray (1994). ${ }^{11}$ It suffices here to focus on the squared term, which imputes a large weight to

\footnotetext{
${ }^{9}$ See also Collier and Hoeffler (1998), Alesina, Baqir and Easterly (1999), Ellingsen (2000), Hegre et al. (2001), Alesina et al. (2003) and Fearon (2003) among others.

${ }^{10}$ Foster and Wolfson (1992) and Wolfson $(1994,1997)$ proposed an alternative measure of polarization specifically designed to capture the "disapearence of the middle class". Later, alternative measures of polarization have been proposed by Wang and Tsui (2000), Chakravarty and Majumder (2001), Zhang and Kanbur (2001), Reynal-Querol (2002), Rodríguez and Salas (2002), and Esteban, Gradín and Ray (2007).

${ }^{11}$ Although in Esteban and Ray (1994) and Duclos, Esteban and Ray (2004) groups are identified by their income - and hence $\delta_{i j}$ is the income distance between the two groups - the notion and measure
} 
group identification. This weighting of group size implies that $P$ does not satisfy Dalton's Transfer Principle (or equivalently, compatibility with second-order stochastic dominance of distance distributions). In this fundamental aspect it behaves differently from Lorenz-consistent inequality measures. In particular, $P$ attains its maximum at a symmetric bimodal distribution.

As in the case of fractionalization, a situation of particular relevance is one in which group distances are binary: $\delta_{i j}=1$ for all $j \neq i$ and $\delta_{i i}=0$. In this case $P$ reduces to

$$
\tilde{P}=\sum_{i=1}^{m} n_{i}^{2}\left(1-n_{i}\right)
$$

This is the measure of polarization proposed by Reynal-Querol (2002).

\section{A Model of Conflict}

We wish to explore the relationship between the measures described in the previous section and the equilibrium level of conflict attained in a behavioral model in which agents optimally choose the amount of resources to expend in conflict. ${ }^{12}$

3.1. Public and Private Goods. Consider a society composed of individuals situated in $m$ groups. Let $N_{i}$ be the number of individuals in group $i$, and $N$ the total number of individuals, so that $\sum_{i=1}^{m} N_{i}=N$. These groups are assumed to contest a budget with per capita value normalized to unity. We shall suppose that a fraction $\lambda$ of this budget is available to produce society-wide public goods. One of the groups will get to control the mix of public goods (as described below), but it is assumed that $\lambda$ is

of polarization can be naturally adapted to the case of "social polarization". Duclos, Esteban and Ray (2004) consider the case of "pure social polarization", in which income plays no role in group identity or inter-group alienation. For that case they propose (4) as the appropriate polarization measure (pp. 1759) with $\delta_{i j}$ interpreted as the alienation felt by an individual of group $i$ with respect to a member of group $j$.

${ }^{12}$ We build on the model of conflict in Esteban and Ray (1999). 
given. The remaining fraction, $1-\lambda$, can be privately divided, and once again the "winning" group can seize these resources. ${ }^{13}$

All individuals derive identical linear payoff from their consumption of the private good, but differ in their preference over the public goods available. All the members of a group share the same preferences. Each group has a mix of public goods they prefer most. Using the private good as numeraire, define $u_{i j}$ to be public goods payoff to a member of group $i$ if a single unit per-capita of the optimal mix for group $j$ is produced. We may then write the per capita payoff to group $i$ as $\lambda u_{i i}+(1-\lambda)\left(N / N_{i}\right)$ (in case $i$ wins the conflict) and $\lambda u_{i j}$ (in case some other group $j$ wins). ${ }^{14}$

The parameter $\lambda$ can also be interpreted as an indicator of the importance of the public good payoff relative to the "monetary" payoff used as numeraire.

We presume throughout that $u_{i i}>u_{i j}$ for all $i, j$ with $i \neq j$. These payoff differences define a natural notion of "distance" or "alienation" across groups: $\delta_{i j} \equiv u_{i i}-u_{i j}{ }^{15}$

3.2. Conflict Resources and Outcomes. We view conflict as a situation in which there is no agreed-upon rule aggregating the alternative claims of different groups. The success of each group is taken to be probabilistic, depending on the expenditure of "conflict resources" by the members of each group. We now describe this conflict.

Let $r$ denote the resources expended by a typical member of any group. We take such expenditure to involve a cost of $c(r)$, and assume

\footnotetext{
${ }^{13}$ This description may correspond to a conflict for the control of the government. Once in government the group may decide to change the types of public goods provided and the beneficiaries of the various forms of transfers in the budget. But it is not possible to substantially modify the structure of the budget.

${ }^{14}$ Note that there is no exclusion in the provision of public goods. These are always provided to the entire population; only the mix differs depending on which group has control. The implicit assumption is that a scaling of the population requires a similar scaling of public goods output in order to generate the same per-capita payoff. Because we hold the per-capita budget constant (and therefore change total budget with population), this gives us exactly the specification in the main text.

${ }^{15}$ While our main result does not presume symmetry $-\delta_{i j}=\delta_{j i}$ for all $i$ and $j-$ the subsequent checks on accuracy do assume it. We do not impose the triangle inequality, however.
} 
[C] $c(r)$ is thrice differentiable with $c(0)=c^{\prime}(0)=0, c^{\prime}(r)>0$ and $c^{\prime \prime}(r)>0$ for all $r>0$, and $c^{\prime \prime \prime}(r) \geq 0$ for all $r \geq 0$.

Condition [C] is standard except for its restriction on the third derivative of $c$. This condition will be used to guarantee the uniqueness of conflict equilibrium. ${ }^{16}$ A special case of interest is isoelastic cost: $c(r)=(1 / \theta) r^{\theta}$. Condition $\mathrm{C}$ is satisfied if $\theta \geq 2$.

Denote by $r_{i}(k)$ the contribution of resources by member $k$ of group $i$, and define $R_{i} \equiv \sum_{k \in i} r_{i}(k)$. Our measure of societal conflict is the total of all resources supplied by every individual:

$$
R=\sum_{i=1}^{m} R_{i} .
$$

Let $p_{i}$ be the probability that group $i$ wins the conflict. We suppose that

$$
p_{i}=\frac{R_{i}}{R}
$$

for all $i=1, \ldots, m$, provided that $R>0 .{ }^{17}$ Thus the probability that group $i$ will win the lottery is taken to be exactly equal to the share of total resources expended in support of alternative $i$.

3.3. Payoffs and Extended Utility. We may therefore summarize the overall expected payoff to an individual $k$ in group $i$ as

$$
\begin{aligned}
\pi_{i}(k) & =\sum_{j=1}^{m} p_{j} \lambda u_{i j}+p_{i} \frac{(1-\lambda) N}{N_{i}}-c\left(r_{i}(k)\right) \\
& =\sum_{j=1}^{m} p_{j} \lambda u_{i j}+p_{i} \frac{(1-\lambda)}{n_{i}}-c\left(r_{i}(k)\right),
\end{aligned}
$$

where $n_{i} \equiv N_{i} / N$ is the population share of group $i$.

\footnotetext{
${ }^{16}$ While the current model is more general, the argument in Esteban and Ray (1999) can be applied here, as can their counterexample to uniqueness when the condition on $c^{\prime \prime \prime}$ fails.

${ }^{17}$ Assign some arbitrary vector of probabilities (summing to one) in case $R=0$. There is, of course, no way to complete the specification of the model at $R=0$ while maintaining continuity of payoffs for all groups. So the game thus defined must have discontinuous payoffs. This poses no problem for existence; see Esteban and Ray (1999).
} 
We now turn to a central issue: how are resources chosen? For reasons that will become clear, we wish to allow for a flexible specification in which (at one end) individuals choose $r$ to maximize their own payoff, while (at the other end) there is full intra-group cohesion and individual contributions are chosen to maximize group payoffs. We permit these cases as well as a variety of situations in between by defining a group- $i$ member $k$ 's extended utility to be

$$
U_{i}(k) \equiv(1-\alpha) \pi_{i}(k)+\alpha \sum_{\ell \in i} \pi_{i}(\ell)
$$

where $\alpha$ lies between 0 and 1 . When $\alpha=0$, individual payoffs are maximized. When $\alpha=1$, group payoffs are maximized. Note that $k$ enters again in the summation term in (9), so the weight on own payoffs is always 1.

We are open to various interpretations of $\alpha .{ }^{18}$ It could represent altruism, as in the study of cooperatives by Sen (1966), or in models of voting behavior (see, e.g., Edlin et al. (2007), Fowler (2006) and Evren (2009)), or in intergenerational models (Barro and Becker (1989)). But this is not the only possible interpretation. An equivalent (but somewhat looser) view is that $\alpha$ is some reduced-form measure of the extent to which within-group monitoring, along with promises and threats, manages to overcome the free-rider problem of individual contributions. One way to formalize this is to suppose that a "group leader" has as her goal the maximization of the utilitarian objective $\sum_{\ell \in i} \pi_{i}(\ell)$, while a particular individual $k$ is simply concerned with the selfish objective $\pi_{i}(k)$. A compromise is achieved through bargaining and negotiation, resulting in the convex combination described in (9). In this case $\alpha$ may be viewed as the "bargaining power" of the group leader. For a similar shorthand in a different context, see Grossman and Helpman (1994) and Epstein and Nitzan (2002). ${ }^{19}$

\footnotetext{
${ }^{18}$ However, it will not matter whether extended utility is defined on other individual's payoffs (the specification here), or their gross expected payoff excluding resource cost, or indeed on others' extended utility. (In this last case we would need a contraction property for extended utility to be well-defined.) The results are insensitive to the exact choice.

${ }^{19}$ In these models, the payoff to a government is effectively a convex combination of social welfare and the payoff to special interest groups, but not because the government intrinsically cares about those groups.
} 
We are comfortable with either interpretation, but formally take it that each individual acts to maximize the expectation of extended utility, as defined in (9).

3.4. Equilibrium. The choice problem faced by a typical individual member $k$ of group $i$ is easy to describe: given the vector of resources expended by all other groups and by the rest of the members of the own group, choose $r_{i}(k)$ to maximize (9). This problem is well-defined provided that at least one individual in at least one other group expends a positive quantity of resources.

Some obvious manipulation shows that the maximization of (9) is equivalent to the maximization of

$$
\left[(1-\alpha)+\alpha N_{i}\right]\left[p_{i} \frac{1-\lambda}{n_{i}}+\lambda \sum_{j=1}^{m} p_{j} u_{i j}\right]-c\left(r_{i}(k)\right)-\alpha \sum_{\ell \in i ; \ell \neq k} c\left(r_{i}(\ell)\right)
$$

by the choice of $r_{i}(k)$. To write this expression more conveniently, recall our definition of "distance" from $i$ to $j: \delta_{i j} \equiv u_{i i}-u_{i j}$. Now define (for every $i$ and $j$ ) $\Delta_{i i} \equiv 0$, and $\Delta_{i j} \equiv \lambda \delta_{i j}+(1-\lambda) / n_{i}$ for all $j \neq i$, and let $\sigma_{i} \equiv(1-\alpha)+\alpha N_{i}$. Then our individual equivalently chooses $r_{i}(k)$ to maximize

$$
-\sigma_{i} \sum_{j=1}^{m} p_{j} \Delta_{i j}-c\left(r_{i}(k)\right)
$$

Provisionally assuming that $r_{j}(\ell)>0$ for some $\ell \in j \neq i$, the solution to the choice of $r_{i}(k)$ is completely described by the interior first-order condition:

$$
\frac{\sigma_{i}}{R} \sum_{j=1}^{m} p_{j} \Delta_{i j}=c^{\prime}\left(r_{i}(k)\right)
$$

where we use (6) and (7).

An equilibrium is a collection $\left\{r_{i}(k)\right\}$ of individual contributions where for every group $i$ and member $k, r_{i}(k)$ maximizes (10), given all the other contributions. ${ }^{20}$

Proposition 1. An equilibrium always exists and it is unique. In an equilibrium, every individual contribution satisfies the first-order condition (11). In particular, in every group, members make the same contribution: $r_{i}(k)=r_{i}(\ell)$ for every $i$ and $k, \ell \in i$.

\footnotetext{
${ }^{20}$ This is exactly a Nash equilibrium, though we simply refer to "equilibrium" in what follows.
} 
Proof. First observe that in any equilibrium, $R_{j}>0$ for some group $j .{ }^{21}$ But this means that every member of every group other than $j$ must satisfy (11). This proves that in equilibrium, $R_{i}>0$ for all $i$, and that for every group $i$ and $k \in i$, (11) is satisfied. In particular, we see that $r_{i}(k)=r_{i}(\ell)$ for every $i$ and $k, \ell \in i$.

Call this common value $r_{i}$. Multiply both sides of (11) by $r_{i}$ and use (7) to see that

$$
\frac{\sigma_{i}}{N_{i}} \sum_{j=1}^{m} p_{i} p_{j} \Delta_{i j}=r_{i} c^{\prime}\left(r_{i}\right),
$$

and now define $v_{i j} \equiv \sigma_{i} \Delta_{i j} / N_{i}$ for all $i$ to obtain the system

$$
\sum_{j=1}^{m} p_{i} p_{j} v_{i j}=r_{i} c^{\prime}\left(r_{i}\right)
$$

for all $i$. This is precisely the system described in Proposition 3.1 of Esteban and Ray (1999), with $s$ in place of $p$. Under [C], the proof of Proposition 3.2 applies entirely unchanged to show that the system (12) has a unique solution.

When the cost function is quadratic (isoelastic with $\theta=2$ ), we can express the equilibrium of the conflict game in particularly crisp form. For each $i$, the equilibrium condition (12) can now be written as

$$
\sum_{j=1}^{m} p_{j} v_{i j} n_{i}^{2}=p_{i} \rho^{2},
$$

where $\rho \equiv R / N$ is "per-capita conflict". Denote by $W$ the $m \times m$ matrix with $n_{i}^{2} v_{i j}$ as representative element. Then the equilibrium probability vector $\mathbf{p}$ and per-capita conflict level $\rho$ must together solve

$$
W \mathbf{p}=\rho^{2} \mathbf{p},
$$

so that $\rho^{2}$ is the unique positive eigenvalue of the matrix $W$ and the equilibrium vector of win probabilities $\mathbf{p}$ is the associated eigenvector on the $m$-dimensional unit simplex.

\footnotetext{
${ }^{21}$ If this is false, then $R_{i}=0$ for all $i$ so that each group has a success probability given by the arbitrary probability vector specified in footnote 17 . For at least one group, say $j$, this probability must be strictly less than one. But any member of $j$ can raise this probability to 1 but contributing an infinitesimal quantity of resources, a contradiction.
} 


\section{Polarization, Inequality AND CONFlict}

In this section, we introduce our central formula, one that links equilibrium conflict to a linear combination of the distributional measures discussed earlier. The formula is not an exact description of the "true" equilibrium outcome, but the remainder of the paper will argue that it yields a good approximation.

Let $\gamma_{i}$ stand for the ratio of group $i^{\prime}$ s win probabilities $p_{i}$ to its population share $n_{i}$ : $\gamma_{i} \equiv p_{i} / n_{i}$. This variable captures the deviation of win probability from population share that is created by the equilibrium variation of individual effort $r$ across groups. If there were no such differences across groups, win probabilities would simply equal group population shares. Thus the $\gamma_{i}$ 's may be thought of as "behavioral correction factors".

Let us see how the correction factors enter into the determination of per-capita equilibrium conflict. Recall the equilibrium condition (11) with $r_{i}(k)=r_{i}$ for all $k \in i$, as asserted in Proposition 1:

$$
\frac{\sigma_{i}}{R} \sum_{j=1}^{m} p_{j} \Delta_{i j}=c^{\prime}\left(r_{i}\right)
$$

Multiply both sides of this equation by $\rho p_{i}$ and use $\gamma_{i}=p_{i} / n_{i}$ to obtain, for each $i$ :

$$
\sum_{j=1}^{m} \gamma_{i} \gamma_{j} n_{i} n_{j} \frac{\sigma_{i} \Delta_{i j}}{N}=\rho p_{i} c^{\prime}\left(\gamma_{i} \rho\right) .
$$

Multiplying both sides of (14) by $c^{\prime}(\rho) / c^{\prime}\left(\gamma_{i} \rho\right)$, and summing over all $i$, we have

$$
\sum_{i=1}^{m} \sum_{j=1}^{m} \phi\left(\gamma_{i}, \gamma_{j}, \rho\right) n_{i} n_{j} \frac{\sigma_{i} \Delta_{i j}}{N}=\rho \sum_{i=1}^{m} p_{i} c^{\prime}(\rho)=\rho c^{\prime}(\rho),
$$

where

$$
\phi\left(\gamma_{i}, \gamma_{j}, \rho\right) \equiv \frac{c^{\prime}(\rho) \gamma_{i} \gamma_{j}}{c^{\prime}\left(\gamma_{i} \rho\right)} .
$$

Define a unique solution $\hat{\rho}$ to the identity

$$
\hat{\rho} c^{\prime}(\hat{\rho}) \equiv \sum_{i=1}^{m} \sum_{j=1}^{m} n_{i} n_{j} \frac{\sigma_{i} \Delta_{i j}}{N}
$$

Now notice from (16) that $\phi\left(\gamma_{i}, \gamma_{j}, \rho\right)=1$ if $\gamma_{i}$ and $\gamma_{j}$ are both set equal to 1 . With this in mind, we say that $\hat{\rho}$, as defined in (17), is a shorthand for the value of per-capita equilibrium conflict, as obtained in (15). This yields 
PROPOSITION 2. Suppose that all behavioral correction factors are neglected; i.e., set equal to 1. Then the resulting shorthand for equilibrium per-capita conflict is determined by a combination of the three distributional measures $G, P$ and $F$ as follows:

$$
\hat{\rho} c^{\prime}(\hat{\rho})=\omega_{1}+\omega_{2} G+\alpha[\lambda P+(1-\lambda) F]
$$

where $\omega_{1} \equiv(1-\lambda)(1-\alpha)(m-1) / N, \omega_{2} \equiv \lambda(1-\alpha) / N$, and the "distances" used in $G$ and $P$ are precisely the public-goods utility losses $\delta_{i j}=u_{i i}-u_{i j}$.

In particular, when population is large, the shorthand $\hat{\rho}$ is determined by a convex combination of only $P$ and $F$, provided that group cohesion $\alpha>0$.

Proof. Given the discussion up to (15) and (16), it only remains to prove that the identity (17) yields (18). This is a matter of direct inspection by unpacking the $\sigma_{i}{ }^{\prime}$ s and the $\Delta_{i j}$ 's in (17).

The proposition states that provided we can neglect the behavioral correction factors in the way described above, the theory yields a remarkably spare description of conflict. To be sure, barring special cases, we cannot neglect the correction factors and hope for an exact equality of shorthand and true value. However, (a) for the remainder of this section we will treat the shorthand as a good approximation and explain the insights that it provides, and (b) in Section 5 we will discuss the ways in which in fact the approximation is a good one.

The proposition asserts that equilibrium conflict $\rho$ is "approximately" the same as a shorthand measure $\hat{\rho}$, and provides the formula in (18) for the shorthand. The lefthand side of (18) is a strictly monotone function of $\hat{\rho}$; on the right-hand side is a simple linear combination of three familiar distributional indices; the Gini coefficient, the Herfindahl-Hirschman fractionalization index, and the Esteban-Ray polarization measure with coefficient $\beta=1$ (see (4)).

Moreover, the weights on the combination tell us when each measure is likely to be a more important covariate of conflict. Specifically, the weights associated to each of these three indices depend on the degree of publicness of the prize, as captured by $\lambda$, and on the level of intra-group cohesion, as described by $\alpha$. They also depend on overall population. 
The formula simplifies further in an important special case. As population grows large, the weight on the "intercept term" as well as the Gini coefficient converges to zero. Conflict is proxied by a convex combination of polarization and fractionalization, no matter what the value of cohesion, as long as the latter is positive. Thus, as long as we believe that population size is "large", the existence of group cohesion matters, but not its extent.

More intuition and discussion can be found in Sections 5.2, where we conduct some numerical exercises, and in Section 6, where we discuss some extensions of our results.

The merit of a decomposition such as (18) depends on whether it yields a deeper and more intuitive understanding of the factors influencing conflict beyond the abstractions of a specific model. We would claim that our decomposition does accomplish this to some degree. It seems reasonable to classify the main forces driving conflict into three categories: group size, group objectives (public versus private prizes) and group ability to circumvent the free-rider problem ("cohesion" or "identification"). These are precisely the ingredients emphasized in Esteban and Ray (1994) and Duclos, Ray and Esteban (2004) as the main determinants of conflict.

Suppose that we observe a situation of conflict in which all groups fight for the control of an excludable private good (such as the revenue from valuable natural resources). Then the only feature distinguishing the different groups is their size. There is no "primordial" inter-group alienation relevant to this conflict. In that case we should expect that the distribution of group sizes will be the most relevant explanatory factor for conflict. Any measure designed to capture inter-group "distances" should have little to say here. Indeed, the decomposition above with full privateness $-\lambda=0-$ leaves group fractionalization as the sole relevant indicator for conflict.

At the other extreme, full publicness brings out the natural differences in group preferences over public goods. Now fractionalization plays no role, and only the measures reflecting inter-group alienation remain: the Gini and the polarization index.

In the special case of pure contests, individuals make no distinction between the different mixes of public goods chosen by the rivals, so that $u_{i j}$ is independent of $j$ for every group $i$. In this case, $P$ reduces to $\tilde{P}$, the specific polarization measure defined 
in (5). Moreover, $G$ reduces to a multiple of $F .^{22}$ We are therefore left with just $F$ and $\tilde{P}$ as correlates of conflict. ${ }^{23}$ As is well known, these two indices, in spite of their apparent similarity, behave quite differently. $F$ is the sum of a concave transformation of the $n_{i}$ and hence increases with the equalization of population sizes among any subset of groups. In contrast, $\tilde{P}$ is the sum of functions of $n_{i}$ that are convex for low values of $n$ - certainly for $n_{i} \leq 1 / 3-$ and concave for higher values, around $1 / 2$. Hence, the equalization of the sizes of small groups will decrease polarization, while the change is the opposite if we equalize the size of large groups. Therefore, the behavior of $F$ and $\tilde{P}$ in this approximation is significantly different.

In summary, what is remarkable about Proposition 2 is that three measures - and only these three - are highlighted by our model of conflict. It is the simplicity of this relationship which is the main contribution of the paper.

However, we reiterate that this extremely simple structure depends on the (seemingly arbitrary) restriction that all behavioral correction factors equal unity. We now judge the accuracy of our shorthand by studying the exact solution for conflict and comparing this with the approximate solution described in Proposition 2.

\section{ACCURACY OF THE SHORTHAND}

Our exercise would be seriously incomplete if we did not examine the accuracy of our formula. Not only will behavioral correction factors generally depart from unity, there are questions for which this departure is of first order interest. For example, Esteban and Ray (1999) study the "activism" of "extremist" groups (those that are positioned at one end of a line in preference space), defining activism precisely by the ratio of $p_{i}$ to $n_{i}$. Or consider the well-known Pareto-Olson thesis, which argues that small groups have a higher ratio of $p_{i}$ to $n_{i}$, in part because of the higher per-capita gains at stake. These are important issues in their own context, and indeed our model exhibits these features, but they are not under consideration here. So it is legitimate

\footnotetext{
${ }^{22}$ In this case, $\delta_{i j}=\delta$ for all $i \neq j$. Setting $\delta=1$ involves an additional normalization which can be absorbed by adjusting the value of $\lambda$.

${ }^{23}$ However, $F$ enters "twice", once by itself and once as a special case of the Gini. As the discussion in Section 6 reveals, these two entries stem from different forces.
} 
to ask whether sacrificing the correction factors can significantly alter the structural relationship asserted in Proposition $2 .^{24}$

We approach this question from different angles. First, we explore the most restrictive case, providing conditions under which every correction factor equals one. As we've mentioned, an accurate shorthand does not require such a strong restriction, so we turn next to numerical simulations that establish correlations between equilibrium conflict and our shorthand. Finally, we establish a general analytical result on accuracy for the case of high conflict.

5.1. When Are Correction Factors All Equal to Unity? There is a restrictive subclass of models for which all correction factors are unequivocally equal to 1 . In this smaller domain, our formula is exact. It is possible to completely characterize this domain.

Proposition 3. Fix any cost function satisfying Condition C. Then the correction factors $\gamma_{i}$ equal 1 for all $i$ if and only if the eigenvector of the matrix $W$ with representative term $n_{i}^{2} v_{i j}$, as defined in Section 3.4, is $\mathbf{n}$.

Proof. Recall (11), and multiply both sides of this first-order condition by $n_{i} / N$ to obtain

$$
\sum_{j=1}^{m} p_{j} n_{i}\left(\sigma_{i} \Delta_{i j} / N\right)=n_{i} c^{\prime}\left(r_{i}\right) \rho
$$

Using $p_{j}=\gamma_{j} n_{j}, r_{i}=\gamma_{i} \rho$, and $w_{i j}=n_{i}^{2} v_{i j}=n_{i} \sigma_{i} \Delta_{i j} / N$, we see that

$$
\sum_{j=1}^{m} \gamma_{j} n_{j} w_{i}=n_{i} c^{\prime}\left(\gamma_{i} \rho\right) \rho
$$

for $i=1, \ldots, m$.

Clearly, if $\gamma_{i}=1$ for all $i,(19)$ shows right away that $\mathbf{n}$ is the eigenvector of $W$, which establishes necessity. Conversely, if $\mathbf{n}$ is the eigenvector of $W$, then (19) is satisfied by

\footnotetext{
${ }^{24}$ This sort of shorthand is common in economics. For instance, we use GNP as a shorthand of social welfare or the Gini index of the distribution of personal income as a shorthand for the level of equality. In both cases these measures abstract from the effects of endogenous individual choices, such as labor effort or consumption decisions (among other things). Yet, we find them useful indicators for the complex variables they intend to capture. Sacrificing the behavioral correction factors is exactly in the same spirit.
} 
setting all $\gamma_{i}=1$, and $\rho$ such that $c^{\prime}(\rho) \rho$ is the eigenvalue of $W$, and this describes an equilibrium. It is the only one, by the assumed properties of the cost function.

This condition is restrictive; as we have said, correction factors will generally depart from unity. But we can give some simple examples in which these conditions are indeed met.

The first example is one with public goods alone (so that $\lambda=1$ ) and just two groups. Then, provided we also assume that cohesion is perfect $\alpha=1$ or that $N$ is large,

$$
W=\left[\begin{array}{cc}
0 & n_{1}^{2} \delta_{12} \\
n_{2}^{2} \delta_{21} & 0
\end{array}\right]
$$

If the utility loss from the opponent's victory is symmetric across the two groups, then $\delta_{12}=\delta_{21}$, and it is easy to see that the eigenvector condition is necessarily satisfied for any population distribution.

Our second example is one in which all groups are of the same size and the overall utility losses from rival public goods being implemented are the same for all groups: $\sum_{j} \delta_{i j}$ is independent of $i$. (The case of pure contests guarantees the second requirement.) Then, once again, the eigenvector condition is satisfied, independently of the values of $\alpha, \lambda$ or $N$.

These examples do highlight the fact that our condition is not vacuous. But they also reveal just how unlikely it is that the condition will hold in any situation that departs from these special cases. (However, we later discuss a context in which one of these cases acquires particular relevance.) In order to illustrate how restrictive this condition is, extend the first example to three groups. It can now be shown that for every arbitrary preference profile, there is just one strictly positive population vector $\mathbf{n}$ such that the eigenvector condition is satisfied.

We can therefore conclude that barring the first two examples, it is generally an exceptional outcome that $\gamma_{i}=1$ for all $i$. At the same time, it is important to appreciate that this condition is far from necessary to obtain the nearness of the expression

$$
\rho c^{\prime}(\rho)=\sum_{i=1}^{m} \sum_{j=1}^{m} \phi\left(\gamma_{i}, \gamma_{j}, \rho\right) n_{i} n_{j} \frac{\sigma_{i} \Delta_{i j}}{N}
$$


to its counterpart

$$
\hat{\rho} c^{\prime}(\hat{\rho})=\sum_{i=1}^{m} \sum_{j=1}^{m} n_{i} n_{j} \frac{\sigma_{i} \Delta_{i j}}{N},
$$

where $\phi$ is defined in (16). Thus our second approach relies on a numerical examination of this question. Our objective is to see how close $\rho$ is to $\hat{\rho}$ in a variety of simulated exercises.

5.2. Numerical Analysis. We run a series of simulations based on random draws for the parameter values describing group sizes and preferences. For each of these randomly drawn societies we compute the exact level of conflict $\rho$, and compare it to our linear approximation $\hat{\rho}$, given by

$$
\hat{\rho} c^{\prime}(\hat{\rho})=\omega_{1}+\omega_{2} G+\alpha[\lambda P+(1-\lambda) F]
$$

where $\omega_{1}$ and $\omega_{2}$ are as described in Proposition 2. We want to verify how closely our approximation $\hat{\rho}$ is correlated with the true value $\rho$. To cut down on the number of possibilities, we presume that costs come from the isoelastic class $c(r)=(1 / \theta) r^{\theta}$, for $\theta \geq 2$.

We also focus (though not exclusively) on the case of large populations. This means that we can discard from our shorthand the constant term and the Gini index. Interestingly enough, controlling for population distribution across groups, we can also discard $\alpha$ as long as it is positive. Study the condition (12) with constant elasticity, using the observation that $\left(\sigma_{i} / N_{i}\right) \approx \alpha$ for $N$ large. Then each $r_{i}^{\theta}$ becomes roughly proportional to $\alpha$, while the equilibrium win probabilities become insensitive to it. We can conclude that for large populations, $\rho^{\theta}$ is roughly proportional to $\alpha$.

Meanwhile, the same is true of our approximation, which states that

$$
\rho^{\theta} \approx \alpha[\lambda P+(1-\lambda F)]
$$

for large $N$. It follows that the relative accuracy of our approximation is independent of $\alpha$ when the population is large (as long as $\alpha$ is positive). This is why in the simulations below we shall fix $\alpha$ at one positive value (say 0.5 ) in the case of large populations. The specific value of $\alpha$ will matter, however, when population is "small". 
5.2.1. A Baseline Case. Our baseline exercise is the case of large populations and contests with quadratic costs, so that the elasticity $\theta$ equals 2 . By the discussion above we take $\alpha=0.5$. We examine several degrees of publicness in the payoffs: $\lambda=0,0.2,0.8$ and 1.0 (we report on $\lambda=0.5$ in a later variation). In each of these cases, we take numerous random draws of a population distribution over five groups.

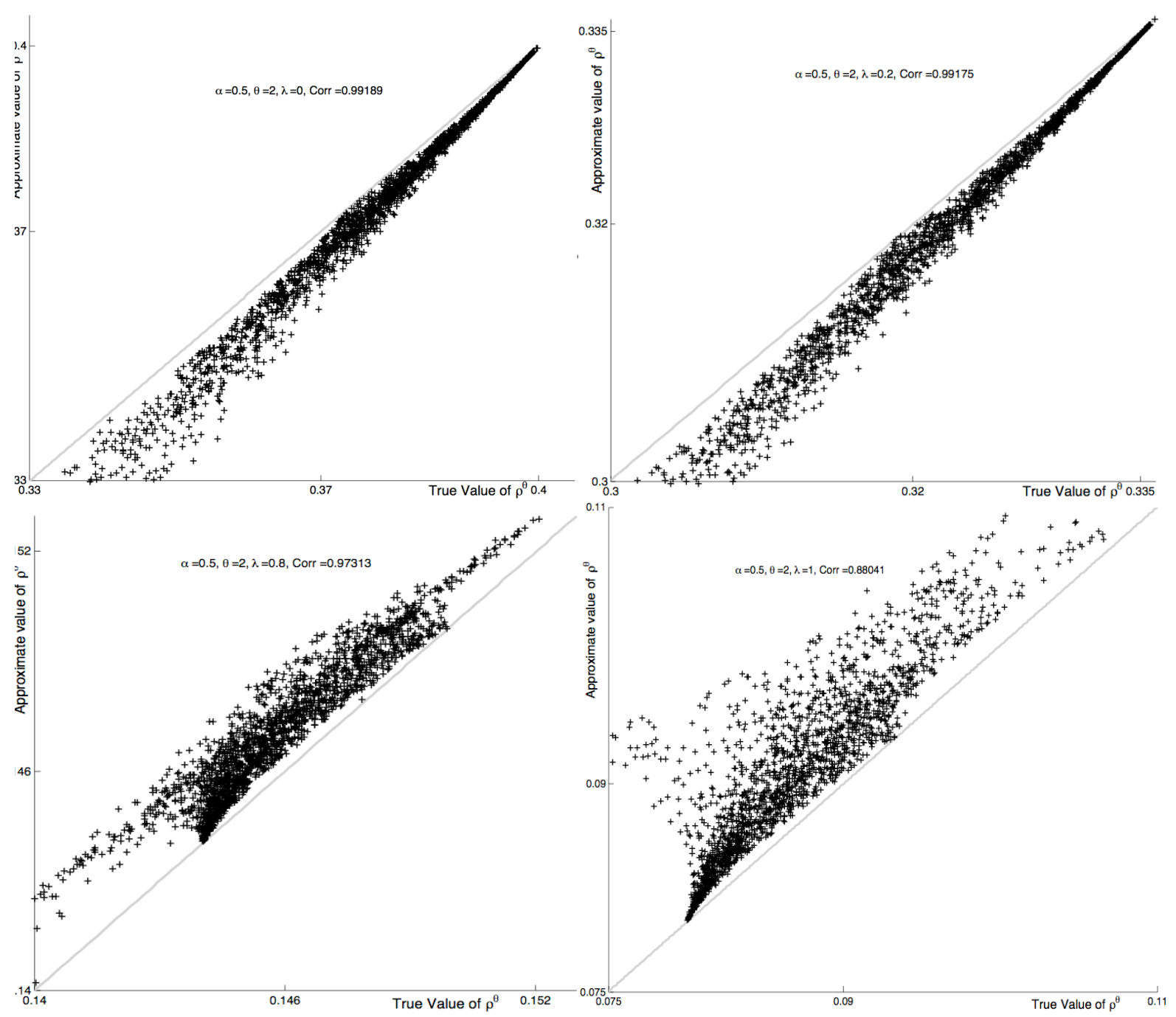

Figure 1. Approximate AND TRUe Conflict: BASEline CASE

Notice that in this case, $\rho c^{\prime}(\rho)=\rho^{2}$. Figure 1 depicts the scatter plot of the approximate and true values of $\rho^{2}$. In each situation (and in all successive figures as well), we plot the true value of conflict on the horizontal axis and the approximation on the vertical axis. We also use the same units for both values, so that the diagonal, shown in every figure, is interpretable as equality in the two values. The top two panels perform 
simulations when private goods are dominant $(\lambda=0.0,0.2)$ and the bottom panels do the same when public goods are dominant $(\lambda=0.8,1.0)$.

We obtain a very strong correlation between the true and the shorthand values for equilibrium conflict suggesting that the "behavior correction factors" do not play a critical role in explaining conflict.

Notice how our formula understates the true value of conflict when $\lambda$ is small, and overstates it when $\lambda$ is close to 1 . There is a good reason for these biases and it is connected to the Pareto-Olson argument. When the conflict is over private goods (which is the case with $\lambda$ small), small groups will put in more resources per-capita, and large groups will put in less. This means that society is really more "polarized" than a measure based on group numbers alone would suggest. Because our approximation ignores this effect, it underestimates conflict. By a converse argument, we tend to overestimate conflict (for small values of conflict) when $\lambda$ is close to 1 . With public goods at stake, small groups put in less resources compared to their population share, and large groups put in more. Therefore society is less "polarized" than what the population numbers alone suggest, and our measure overstates the extent of conflict. Because this observation is not central to our paper, we omit a more detailed discussion.

Over- or under-statement apart, the correlation between the two variables is unaffected and the relationship appears broadly linear. What is remarkable is how close the relationship is, and yet how difficult it appears to be to get a full handle on this analytically. That there is no simple relationship between the two values is evident from the highly nontrivial (yet concentrated) scatter generated by the model. One feature that merits particular comment - and that persists through all the variations we later consider - is that the accuracy of our formula improves quite dramatically at high levels of conflict. This phenomenon is closely related to the characterization result of Proposition 3. It provides strong support for our restriction on correction factors. We back this up analytically in the next section (5.3).

A high correlation between $\rho$ and $\hat{\rho}$ is retained in all the reasonable variations that we have studied. Some examples follow. 


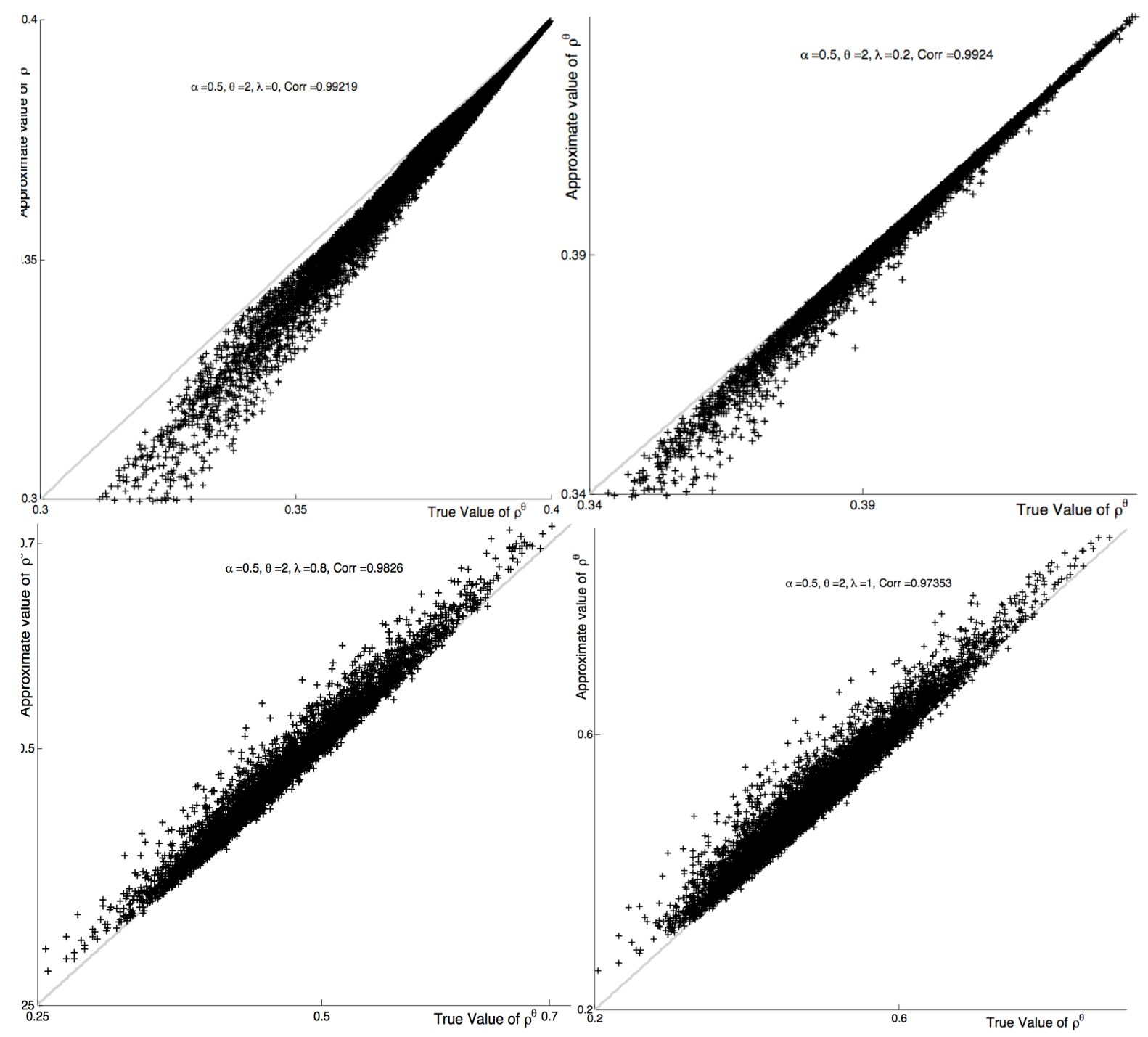

Figure 2. Approximate and TRUe Conflict: VARYing Utility Distances

5.2.2. Inter-Group Distances. The next set of simulations studies varying inter-group distances, instead of pure contests. Recall that distances are to be interpreted as losses from having the other public goods in place, instead of the group's favorite. We modify the previous simulation and now permit utility losses to vary across groups pairs (retaining the symmetry restriction that $u_{i j}=u_{j i}$ ). This is done by taking numerous independent draws of the matrix describing pairwise utility losses. We retain the baseline specification in all other ways. The results are reported in Figure 2, for various values of $\lambda$. As in the baseline case, the top panels perform simulations with private goods $(\lambda=0.0,0.2)$ and the bottom panels do the same for public goods $(\lambda=0.8,1.0)$. 
The correlations continue to be extremely high and the general features of the baseline case are retained.

5.2.3. Small Populations. We return to the case of contests (with quadratic cost functions) and now study "small" populations so that the Gini index can have a role. We suppose that there are 50 individuals in the economy, and consider numerous allocations of this population to the five groups. We report results for one case in which private goods are dominant $(\lambda=0.2)$ and another in which public goods are dominant $(\lambda=0.8)$. Notice that with small populations, the value of group cohesion $\alpha$ will generally matter. The top panel of Figure 3 reports our results for private goods under two values of $\alpha, 0.5$ and 1.0. The bottom panel does the same for public goods. As before, the correlations continue to be very high and the other features discussed for the baseline case are retained.

5.2.4. Other Cost Elasticities. Finally, we explore a set of variations in which we change the cost function from quadratic to other isoelastic specifications. We report four sets of results in Figure 4, all for the case with $\lambda=0.5$ and large populations. One is for the baseline quadratic case. The remaining three are for progressively higher elasticities of the cost function: $\theta=3,4$ and 10 .

Once again, the large correlations that we obtain remain undisturbed. Indeed, the simulations suggest that as the elasticity of the cost function goes up, our approximation improves even further. This is intuitive, as a highly curved cost function will lead to greater uniformity in the per-capita contribution of resources, thereby bringing the behavioral correction factors closer to unity.

It is certainly possible to try different combinations of these variations. We have done so, but do not report these results for the sake of brevity. In all the specifications we have tried, the shorthand we use appears to be more than satisfactory.

5.3. An Analytical Result on Accuracy. We return to the observation - borne out in the numerical simulations - that the accuracy of the formula appears to improve for high values of conflict. In a sense, this high accuracy provides strong justification for 


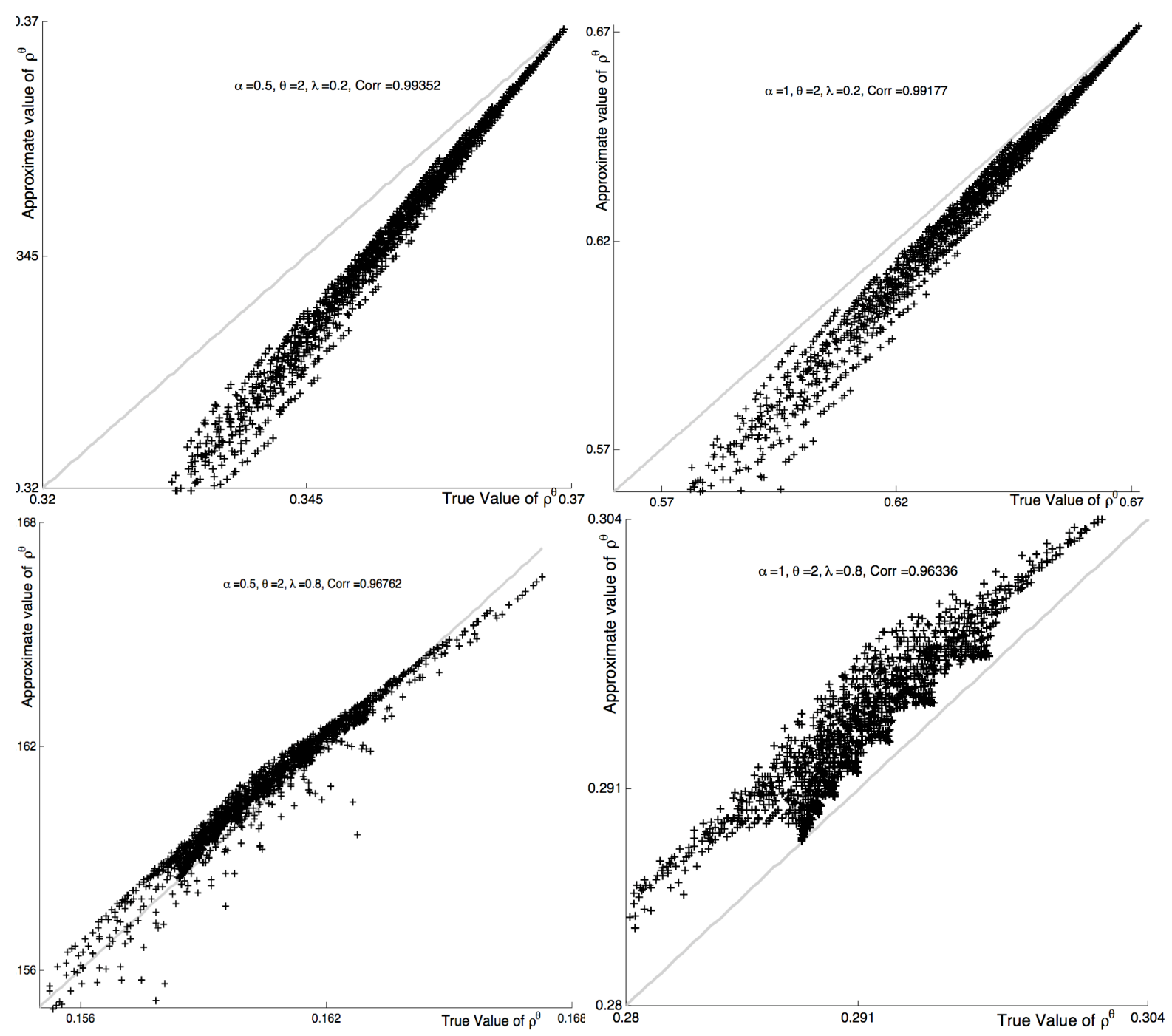

Figure 3. Approximate And True Conflict: Small Population

our restriction on behavioral correction factors. It would therefore be useful to supplement this aspect of the numerical results by an analytical argument. (We agree that it would be even better to analytically establish the high observed correlation between $\rho$ and $\hat{\rho}$ throughout, not just at high levels, but this task will have to be postponed to a later investigation.)

In what follows, we consider the case of "pure contests", in which $\delta_{i j}=1$ for all $i \neq j$. The normalization is without loss of generality as $\lambda$ can be adjusted to capture differences in preference intensities across public and private goods. We also presume - but only for expositional convenience - that $N$ is large enough so that we can think of population shares in each group as a continuous variable. 


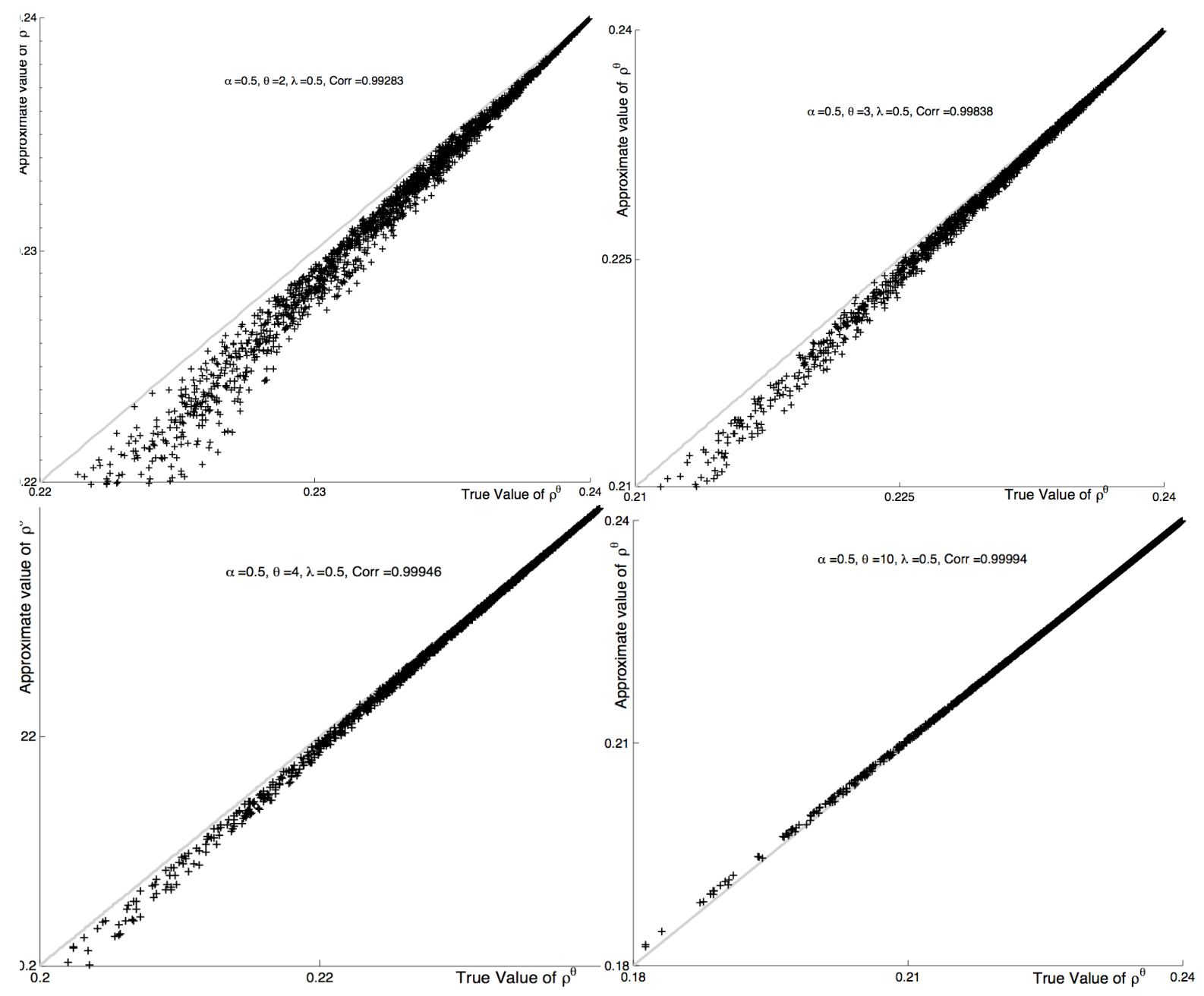

Figure 4. Approximate ANd TRue Conflict: Nonquadratic Costs

Fix group identities and preferences and consider all possible population distributions over the $m$ groups. Then it is easy to see that $\hat{\rho}$ must range over some compact interval $[0, \hat{U}]$.

Proposition 4. Assume pure contests and a large population. Then for every $\epsilon>0$, there exists $\eta>0$ such that whenever $\hat{\rho}>\hat{U}-\eta$,

$$
|\rho-\hat{\rho}|<\epsilon
$$

for every possible value of conflict $\rho$ under a population distribution that generates $\hat{\rho}$.

This proposition shows that the accuracy of our "shorthand" formula becomes extremely high as $\hat{\rho}$ reaches its upper bound. The proof of this result relies on the observation (see Lemma 1 in the Appendix) that at high values of $\hat{\rho}$, the occupied groups 
are of equal size. With that lemma in place, the proof follows quickly from the exact representation conditions established in Proposition 3.

The observation of the lemma is central to the argument (see Appendix for details). When all prizes are public, so that $\lambda=1$, this observation follows from the fact that the Esteban-Ray polarization measure is maximized at a bimodal distribution. When all prizes are private, so that $\lambda=0$, this observation follows from the fact that fractionalization is maximized with equal population in every group. The question implicitly posed by the lemma is whether the common property of population uniformity (over all occupied groups) persists for intermediate values of $\lambda$. As the proof of Lemma 1 shows, this requires a nontrivial and subtle argument. The reason is that our formula does not have any particular curvature with respect to population vectors; it is both locally convex and locally concave in different zones.

We conjecture that our argument extends to all public goods "distances" that are symmetric. However, we do not have an analytical proof of this result (the simulations continue to support high accuracy at high values of $\hat{\rho}$ for such cases).

\section{Discussion}

We have developed a behavioral model of conflict and have shown that the equilibrium level of conflict can be represented by a linear function of inequality, fractionalization and polarization. This function does not represent an exact fit, but numerical simulations together with some analytical observations show that the connection is remarkably strong.

One might legitimately wonder about the generality of the result. How robust is Proposition 2 to perturbations of the underlying model? In this section, we briefly examine how the results might change when we consider different variations on the model.

Let us review the general picture. We describe inter-group conflict over two objects: privately divisible resources, and public goods. Private resources yield a payoff if the group wins, and none otherwise. Public goods payoffs are more complex: a particular 
group will, in general, enjoy different payoffs if different opponents get to implement their favorite mix of public goods. ${ }^{25}$

Irrespective of the particular model used, then, as long as there is some cohesion within a group, the following two points apply:

1. A high component of publicness in payoffs forces inter-group differences in publicgoods preferences to play a bigger role in behavior. Yet, at the same time, the payoff from public goods is not dissipated by group size. This, coupled with the existence of some group cohesion, brings a measure of polarization to the forefront. Therefore the very same features that emphasize inter-group distances also enhances the explanatory power of polarization. This is why an increase in $\lambda$ increases the weight on polarization, and it is also why inter-group distances enter the polarization index. Note that to get polarization to matter, we need both some group cohesion and some publicness (in the prize).

2. In contrast, when payoffs are private, inter-group "distances" are no longer important. With only a concern for private payoffs, inter-group differences are binary: either you are a winner, or you are not. At the same time, private payoffs - which are divided by group size - dwindle as the group gets larger. The importance of group size is thereby reduced, and a measure of fractionalization takes center stage. This is why a decrease in $\lambda$ increases the weight on fractionalization, and it is also why inter-group distances do not enter the fractionalization index. Note that to get fractionalization to matter for large populations, we need both some group cohesion and some privateness (in the prize).

There is an exception to these two points, and this has to do with the possibility that purely selfish motives - quite apart from group cohesion - might also drive resource contributions to public- and private-goods conflicts. However, the effect will vanish as the population grows large. This feature is captured by the point that the Gini

\footnotetext{
${ }^{25}$ One might conceive of a similar possibility for private goods, with a group experiencing different payoffs ("hate", "envy") depending on the identity of the opponent that wins the private resources. But this, by definition, would be classified as a public goods payoff, and $\lambda$ would be suitably defined to incorporate it.
} 
coefficient (with distances) does appear as an explanatory variable in our formula, but with vanishing weight as population size goes to infinity. In short, the Gini with public-goods distances can matter only for "small populations.

The virtue of the model we have developed is that it permits us to neatly uncover these interactions that, we firmly believe, transcend the particular specification we have used.

We remark on some specific extensions.

6.1. Cohesion Depending on Group Objectives. As already discussed, the parameter $\alpha$ captures the degree of group cohesion. In effect, it measures the weight that each individual assigns to the welfare of the group vis-a-vis her own. We have taken $\alpha$ to be exogenous, while in many situations it may well be determined by other model parameters. While endogenous group cohesion is a project in its own right, a few remarks may be useful here.

First, individuals might display different levels of group cohesion, depending on the particular issue at stake. Controlling for the number of people in the group, the extent of group cohesion may well be affected in, say, a civil-rights movement, relative to a tussle for private resources. In short, the nature of the objective the group is fighting for may have an influence on $\alpha$. A second argument is that the size of the group may modify the level of group cohesion, and it is likely (though not inevitable) that the influence will be negative.

Consider the first point: that cohesion might be influenced by the nature of the objective. We explore the simplest case, in which the degree of altruism is equal to the degree of publicness: $\alpha=\lambda$. Note that this modification will just affect the term $\sigma_{i} \Delta_{i j}$ in (14) and hence the shorthand is exactly the same, with the corresponding substitutions. Performing these changes one obtains

$$
\hat{\rho} c^{\prime}(\hat{\rho})=\tilde{\omega}_{1}+\tilde{\omega}_{2} G+\lambda[\lambda P+(1-\lambda) F]
$$

where $\tilde{\omega}_{1} \equiv(1-\lambda)^{2}(m-1) / N$ and $\tilde{\omega}_{2} \equiv \lambda(1-\lambda) / N$. 
Now, full privateness, $\lambda=0$, makes the three indices irrelevant as explanations of conflict. Full publicness, $\lambda=1$ leaves polarization $P$ as the sole essential index. The importance of the Gini and fractionalization indices is maximal at the intermediate value $\lambda=\frac{1}{2}$. Making $\alpha$ endogenous in this particular way has not made significant changes to our exercise.

6.2. Cohesion Depending on Group Size. The second point concerns the systematic dependence of $\alpha$ on group size. Fix $N$, and assume that $\alpha=n^{\epsilon}$, where $\epsilon$ is likely negative, though we do not insist on this. Here again this change simply modifies the term $\sigma_{i}$ in (14) and hence the shorthand continues to be the same, modulo the corresponding substitution. Indeed, performing this substitution one gets

$$
\hat{\rho} c^{\prime}(\hat{\rho})=\sum_{i=1}^{m} \sum_{j \neq i}^{m} n_{i} n_{j}\left[\frac{1-n_{i}^{\epsilon}}{N}+n_{i}^{\epsilon} n_{i}\right]\left[\lambda \delta_{i j}+\frac{1-\lambda}{n_{i}}\right] .
$$

Letting $(1 / N) \approx 0$, we now obtain

$$
\hat{\rho} c^{\prime}(\hat{\rho})=\lambda P(\epsilon)+(1-\lambda) F(\epsilon)
$$

where

$$
P(\epsilon)=\sum_{i=1}^{m} \sum_{j=1}^{m} n_{i}^{2+\epsilon} n_{j} \delta_{i j} \text { and } F(\epsilon)=\sum_{i}\left(1-n_{i}\right) n_{i}^{1+\epsilon}
$$

are the general polarization index in Esteban and Ray (1994) — with the restriction that $1+\epsilon \in[0,1.6]$ - and a variant of the fractionalization index, respectively.

Note that $P(\epsilon)$ satisfies the properties of a polarization measure for discrete distributions as long as the effect of group size on altruism is not too large and positive $(\epsilon<0.6)$ or not too large and negative $(\epsilon>0)$.

As for $F(\epsilon)$, this too becomes a polarization index in the Esteban-Ray (1994) class when $\epsilon>0$. If, however, we agree that $\epsilon$ is generally negative, then $F(\epsilon)$ behaves as a modified fractionalization index. For instance, $F$ is the sum of a concave transformation of the $n$. Hence, given the number of groups $m, F$ is maximized when all the groups have equal size $\frac{1}{m}$. Further, in that case, $F$ increases with the number of groups. It can be readily verified that both properties are shared by $F(\epsilon)$ when $\epsilon \leq 0$, so that we can consider $F(\epsilon)$ as a generalized version of the fractionalization index. 
We can thus conclude that under the assumption that $\alpha$ depends on the population size we still obtain proxies that can be expressed as a function of inequality, fractionalization and polarization, although now we will need to use generalized forms for polarization and fractionalization. Furthermore, we continue to retain the same properties that more publicness increases the relevance of polarization and decreases that of fractionalization.

6.3. Costs of Conflict. We've considered a somewhat sanitized scenario in which conflict affects win probabilities but does not directly destroy social budgets. But it is easy to extend these arguments to a case in which the disposable budget shrinks with more intense conflict. Suppose that the disposable budget is some differentiable nonincreasing function $f(\rho)$ of the extent of conflict. Each agent $k$ in group $i$ then chooses $r_{i}(k)$ to maximize a variant of (10):

$$
-\sigma_{i} \sum_{j=1}^{m} p_{j} \Delta_{i j} f(\rho)-c\left(r_{i}(k)\right)
$$

Exactly the same arguments apply to show that $r_{i}(k)$ has a common value $r_{i}$, described by an analogue of (14):

$$
\left[f(\rho)-\rho f^{\prime}(\rho)\right] \sum_{j=1}^{m} \gamma_{i} \gamma_{j} n_{i} n_{j} \frac{\sigma_{i} \Delta_{i j}}{N}=\rho p_{i} c^{\prime}\left(\gamma_{i} \rho\right) .
$$

Thereafter exactly the same argument leading up to Proposition 2 can be followed, with the only proviso that the left-hand side of (17) will need to be replaced by the strictly increasing function

$$
\frac{\hat{\rho} c^{\prime}(\hat{\rho})}{f(\hat{\rho})-\hat{\rho} f^{\prime}(\hat{\rho})}
$$

instead of just $\hat{\rho} c^{\prime}(\hat{\rho})$. The variable $\hat{\rho}$ is still a shorthand for $\rho$.

6.4. Contest Success Functions. We conclude these robustness tests of the model by examining the implications of departing from the standard specification of the contests success function given by (7). Let us suppose instead that the win probabilities are

$$
p_{i}=\frac{\sum_{k \in i} \vartheta\left(r_{i}(k)\right)}{\sum_{j} \sum_{k \in j} \vartheta\left(r_{j}(k)\right)}
$$


for some $\vartheta$ strictly increasing. Now, individual resources $r$ are assumed to turn into "effective" conflict resources $z$, so that $z=\vartheta(r)$, and this is what determines the win probabilities.

Notice, though, that we can rewrite the utility cost in therms of $z$ instead of $r$. We then

see that individuals choose $z_{i}(k)$ with a utility cost of $\tilde{c}(z)=c\left(\vartheta^{-1}\left(z_{i}(k)\right)\right)$. Then, as long as $\tilde{c}(\cdot)$ satisfies condition [C], an equilibrium exists and it is unique. It is obvious that our previous analysis goes through by reinterpreting $z$ as the choice variable. Consequently, the linear function of the three distributional indices would now approximate the "effective" aggregate resources per capita expended in conflict, $\tilde{\rho}$.

\section{CONCLUding REMARKS}

We have set up a behavioral model of conflict that provides a basis for the use of $F, G$ and/or $P$ as indicators for conflict.

[A] We have shown that the equilibrium level of resources expended in conflict can be approximated by a linear combination of the three indices, using the degree of altruism and of publicness as weights.

[B] The higher is the altruism the more pertinent fractionalization and polarization are in explaining conflict. The higher the degree of publicness the pertinent indices are inequality and polarization.

[C] In simulations we find a very high correlation between our approximation and the true value of per capita conflict. This suggests that the behavior correction factors do not play a critical role.

Most importantly, this paper suggests new key features in explaining conflict: the degree of publicness in the payoff and the level of group cohesion in individual behavior. While ours is not an empirical exercise, it is clear that our results provoke two sorts of approaches to the data. One could take the structural stance of specifying a cost function, so that the object $\rho c^{\prime}(\rho)$ is well-defined, and then use the formula (18) to estimate the implied parameters of publicness, privateness and group cohesion. It should be noted, however, that for large populations, $\alpha$ cannot be separately identified 
from a multiplicative coefficient on the cost function. On the other hand, the ratio of the coefficients on polarization and fractionalization is informative about $\lambda$, the degree of publicness. ${ }^{26}$

Under a less structural view, one can treat (18) in a looser way: as an indicator of which distributional variables might be related to conflict in different situations, and then examining such predictions via reduced-form techniques. We remain agnostic about either approach.

At a more basic level, one might think of two issues in taking this exercise to the data. The first is precisely what one means by "conflict". Most of the literature has identified social conflict with civil war. To this effect, a country is recorded as having conflict whenever the number of deaths goes above a given threshold (see, for example, the studies by Collier and Hoeffler $(1998,2004)$ and Fearon and Laitin (2003)). But social conflict need not manifest itself in civil war alone, and there are various other measures (that incorporate, for instance, strikes, demonstrations, riots, assassinations, political prisoners and the like). Our model should certainly not be seen as an attempt to explain the onset of civil war, and perhaps should not be used in such a context. It may be somewhat better for civil war incidence, but its most satisfactory application should be - data permitting - as a potential explanation for the broader range of conflicts described here.

A second issue of interest is the choice of groups over which the distributional measures are to be defined. Presumably, certain groupings are salient in any particular society, but the model developed here yields no insights regarding the identification of such groupings. The theory here will therefore need to be combined with others that study salience (see, for example, Esteban and Ray (2008)), or one will have to take the leap of committing, at the outset, to the study of certain groupings, such as ethnicity or religion (see, for example, Montalvo and Reynal-Querol (2005)).

\section{REFERENCES}

\footnotetext{
${ }^{26}$ For small populations, in which we have separate information about population size, $\alpha$ can be identified from the relative importance of the coefficients on the Gini and constant terms on the one size, and polarization/fractionalization on the other.
} 
Alesina, A., R. Baqir, And W. Easterly (1999) "Public Goods and Ethnic Divisions", Quarterly Journal of Economics, 114, 1243-1284.

Alesina, A., A. Devleeschauwer, W. Easterly, S. Kurlat and R. Wacziarg (2003) “Fractionalization",Journal of Economic Growth8 155194.

BARRO, R.J. AND G.S. BECKER (1989) “Fertility Choice in a Model of Economic Growth," Econometrica 57, 481-501.

BRockett, C.D (1992) "Measuring Political Violence and Land Inequality in Central America" American Political Science Review 86 (1992), 169-176.

Chakravarty, S.R. And A. Majumder (2001), "Inequality, Polarization and Welfare: Theory and Applications," Australian Economic Papers 40, 1-13.

Collier, P. And A. Hoeffler (1998) "On the Economic Causes of Civil War" Oxford Economic Papers, 50 563-573.

Collier, P. And A. Hoeffler (2004), "Greed and Grievance in Civil War" Oxford Economics Papers 56, 563-595.

Duclos, J-Y., J. Esteban, And D. RAY (2004), “Polarization: Concepts, Measurement, Estimation" Econometrica 72, 1737-1772.

Easterly W. And R. Levine (1997), Africa's Growth Tragedy: Policies and Ethnic Divisions, Quarterly Journal of Economics 111, 1203-1250.

Edlin, A., Gelman, A., AND N. Kaplan (2007), "Voting as a Rational Choice: Why and How People Vote to Improve the Well-Being of Others," Rationality and Society 19, 293-314.

Ellingsten, T. (2000), "Colorful Community or Ethnic Witches' Brew?" Journal of Conflict Resolution 44, 228-249.

Epstein G. And S. Nitzan (2002), “Endogenous Public Policy, Politicization and Welfare, Journal of Public Economic Theory 4, 661-677. 
Esteban, J., C. GRAdín AND D. RAy (2007) An Extension of a Measure of Polarization, with an application to the income distribution of five OECD countries, Journal of Economic Inequality 5, 1-19.

Esteban, J. And D. RAY (1994), “On the Measurement of Polarization” Econometrica 62, 819-852.

Esteban, J. AND D. RAY (1999), “Conflict and Distribution” Journal of Economic Theory $87,379-415$.

EstebAN, J. AND D. RAY (2001), “Free Riding and the Group Size Paradox" American Political Science Review 95, 663-672.

Esteban,J. And D. Ray (2007), “A Model of Ethnic Conflict,” mimeo., Department of Economics, new York University.

Esteban, J. AND D. RAY (2008a), “Polarization, Fractionalization and Conflict," Journal of Peace Research 45, 163-182.

Esteban, J. And D. RAY (2008b), “On the Salience of Ethnic Conflict,” American Economic Review 98 forthcoming.

Esteban, J. AND G. Schneider (2008) Polarization and Conflict: Theoretical and Empirical Issues (Introduction to the special issue), Journal of Peace Research 45, 131141.

Evren, O. (2009), "Altruism, Turnout and Strategic Voting Behavior," mimeo., Department of Economics, New York University.

FEARON, J. (2003), "Ethnic and Cultural Diversity by Country," Journal of Economic Growth 8, 195-222.

FEARON, J. AND D. LAITIN (2003), "Ethnicity, Insurgency, and Civil War," American Political Science Review 97, 75-90.

Foster, J. AND M.C. Wolfson (1992), "Polarization and the Decline of the Middle Class: Canada and the US", Venderbilt University, mimeo.

FOWLER, J. (2006), “Altruism and Turnout," Journal of Politics 68, 674-683. 
Hegre, H., Ellingsen, T., Gates, S. And N. Gledisch (2001), “Towards a Democratic Civil Peace? Democracy, Political Change, and Civil War, 1816-1992,American Political Science Review 95, 33-48.

Hirschman, A. O. (1964), "The Paternity of an Index," American Economic Review 54, 761-762.

Grossman, G.M. And E. Helpman (1994), Protection for Sale, American Economic Review 84, 833-850.

Horowitz, D.L. (1985), Ethnic Groups in Conflict, Berkeley, CA: University of California Press.

LICHBACH, M. I. (1989), “An Evaluation of ‘Does Economic Inequality Breed Political Conflict?' Studies, World Politics 4, 431-470.

MidlarSKI, M.I. (1988), "Rulers and the Ruled: Patterned Inequality and the Onset of Mass Political Violence," American Political Science Review 82, 491-509.

Miguel, E., Satyanath, S. And E. Sergenti (2004), "Economic Shocks and Civil Conflict: An Instrumental Variables Approach," Journal of Political Economy 112, 725753.

Montalvo, J. G. And M. Reynal-Querol (2005a), “Ethnic Polarization, Potential Conflict and Civil War," American Economic Review 95, 796-816.

Montalvo, J. G. And M. Reynal-Querol (2005b), “Ethnic Diversity and economic development" Journal of Development Economics 76, 293-323.

Muller,E.N. AND M.A. Seligson (1987) "Inequality and Insurgency", American Political Science Review 81, 425-451.

Muller, E. N., M.A. Seligson AND H. Fu (1989), “Land inequality and political violence", American Political Science Review 83, 577-586.

NAGEL, J. (1974), "Inequality and discontent: a non-linear hypothesis," World Politics 26, 453-472. 
Reynal-Querol, M. (2002), "Ethnicity, Political Systems, and Civil Wars" Journal of Conflict Resolution 46, 29-54.

RodríGuez, J.G. AND R. SAlas (2002), "Extended Bi-Polarization and Inequality Measures," Research on Economic Inequality 969-84.

Sambanis, N. (2001), "Do Ethnic and Non-Ethnic Civil Wars Have the Same Causes? A Theoretical and Empirical Enquiry (Part 1)" Journal of Conflict Resolution 45, 259-282.

SEN, A. (1966), "Labour Allocation in a Cooperative Enterprise" Review of Economic Studies 33, 361-371.

SEN, A. K. (1973), On Economic Inequality, Clarendon Press, Oxford.

SKaPerdas, S. (1996), “Contest Success Functions,” Economic Theory 7, 283-290.

THON, D. (1982) "An Axiomatization of the Gini Coefficient", Mathematical Social Science 2, 131-143.

WANG, Y.Q. AND K.Y. Tsui (2000), "Polarization Orderings and New Classes of Polarization Indices," Journal of Public Economic Theory 2, 349-363.

Wolfson, M. C. (1994), “When Inequalities Diverge”, American Economic Review Papers and Proceedings 84 353-358.

WOLFSON, M.C. (1997), "Divergent Inequalities: Theory and Empirical Results", Review of Income and Wealth 43, 401-421.

ZHANG, X. AND R. KAnbuR (2001), “What Difference Do Polarisation Measures Make? An Application to China," Journal of Development Studies 37, 85-98.

\section{APPENDIX}

An Axiomatization of the Quadratic Polarization Index. Esteban and Ray (1994) and Duclos, Esteban and Ray (2004) axiomatize the following class of polarization measures. Let population be distributed on $[0, \infty)$ with density $f(x)$. The class is given by

$$
P_{\beta}=K \iint f(x)^{1+\beta} f(y)|x-y| d x d y, \text { for some constant } K>0 \text { and } \beta \in[0.25,1] \text {. }
$$


Axiom 5. Suppose that a distribution consists of three equi-spaced uniform basic densities of sizes $r, p$ and $q$, as shown in Figure 5, each of support $2 \epsilon$. Assume that $p=q+r$. Then there is $\eta>0$ such that if $0<r<\eta$ and $0<\epsilon<\eta$, any uniform transfer of population mass from $r$ to $q$ cannot decrease polarization.

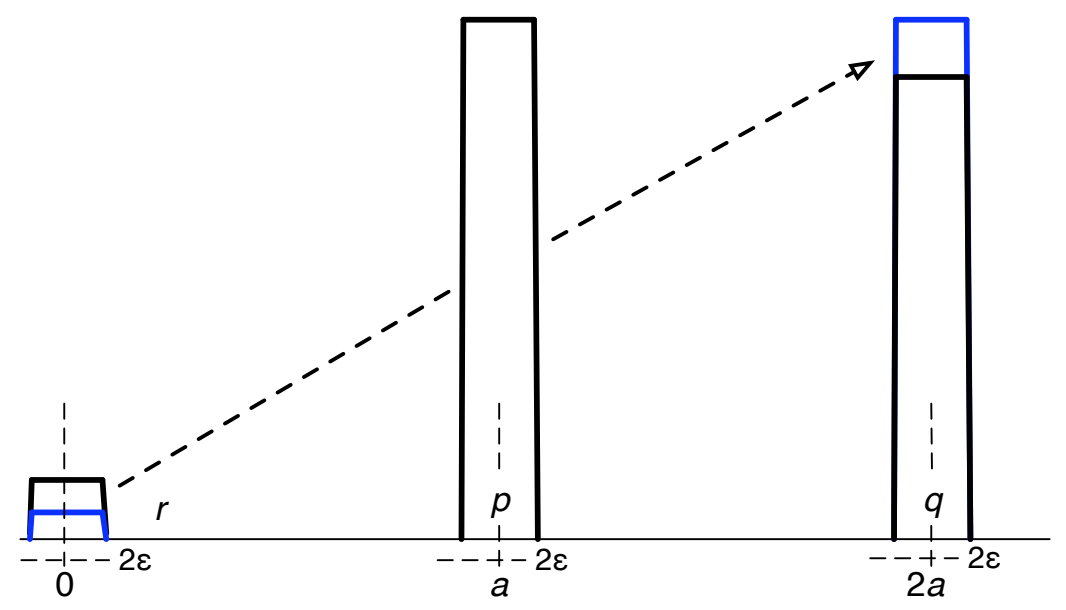

FIGURE 5. Figure for Axiom 5.

Intuitively, this axiom asserts that if the group of size $r$ is extremely small, it cannot be contributing much on its own to social tension. If instead the population is transferred from that group to another group which is "equally opposed" to the largest group of size $p$ (and of mass slightly smaller than $p$ ), then polarization cannot come down.

THEOREM 1. Under the additional Axiom 5, it must be that $\beta=1$, so the unique polarization measure that satisfies the five axioms is proportional to

$$
\iint f(x)^{2} f(y)|y-x| d y d x
$$

Proof. Consider a distribution generated from three copies of a uniform basic density as in Axiom 5, exactly as shown in Figure 5. The bases are centered at locations 0, $a$ and $2 a$. Each has width $2 \epsilon$. The heights are $r, p$ and $q$.

First we show necessity. Suppose that the axiom is true. Take parameters $z \equiv(p, q, r, \epsilon)$ to satisfy the conditions of the axiom, and transfer a small amount $\delta$ uniformly from the $r$-mass to the $q$-mass. Then polarization (viewed as a function of $\delta$ and the other parameters $z$ ) is given by the three "internal" polarizations of each basic density as well as the pairwise effective 
antagonisms across each pair of basic densities, which makes for nine terms in all:

$$
\begin{aligned}
P(\delta, z)= & I(\epsilon)\left[(r-\delta)^{2+\beta}+p^{2+\beta}+(q+\delta)^{2+\beta}\right]+C^{2}(\epsilon)\left[(r-\delta)^{1+\beta}(q+\delta)+(q+\delta)^{1+\beta}(r-\delta)\right] \\
& +C(\epsilon)\left[(r-\delta)^{1+\beta} p+p^{1+\beta}(q+\delta)+(q+\delta)^{1+\beta} p+p^{1+\beta}(r-\delta)\right]
\end{aligned}
$$

where $I(\epsilon)$ is the "total internal distance" within each rectangle:

$$
I(\epsilon) \equiv \int_{-\epsilon}^{\epsilon} \int_{-\epsilon}^{\epsilon}|x-y| d x d y=\frac{8 \epsilon^{3}}{3}
$$

$C(\epsilon)$ is the "total distance" across neighboring rectangles:

$$
C(\epsilon) \equiv \int_{-\epsilon}^{\epsilon} \int_{a-\epsilon}^{a+\epsilon}(x-y) d x d y=4 a \epsilon^{2}
$$

and $C^{2}(\epsilon)$ is the "total distance" between the side rectangles:

$$
C^{2}(\epsilon) \equiv \int_{-\epsilon}^{\epsilon} \int_{2 a-\epsilon}^{2 a+\epsilon}(x-y) d x d y=8 a \epsilon^{2}
$$

Differentiating $P(\delta, z)$ with respect to $\delta$ (write this partial derivative as $P^{\prime}(\delta, z)$ ) and evaluating the result at $\delta=0$, we see that

$$
\begin{aligned}
P^{\prime}(0, z) & =(2+\beta) I(\epsilon)\left[q^{1+\beta}-r^{1+\beta}\right]+(1+\beta) C(\epsilon)\left[q^{\beta} p-r^{\beta} p\right] \\
& -C^{2}(\epsilon)\left[q^{1+\beta}-r^{1+\beta}+(1+\beta)\left(r^{\beta} q-q^{\beta} r\right)\right] .
\end{aligned}
$$

Substituting the values for $I(\epsilon), C(\epsilon)$ and $C^{2}(\epsilon)$, we see that

$$
\begin{aligned}
\frac{1}{4} \epsilon^{-2} P^{\prime}(0, z) & =(2+\beta) \frac{2 \epsilon}{3}\left[q^{1+\beta}-r^{1+\beta}\right]+(1+\beta) a p\left[q^{\beta}-r^{\beta}\right] \\
& -2 a\left[q^{1+\beta}-r^{1+\beta}+(1+\beta)\left(r^{\beta} q-q^{\beta} r\right)\right] .
\end{aligned}
$$

The axiom insists that $P^{\prime}(0, z)$ must be nonnegative for all values of $z$ such that $p=q+r$ and $r$ sufficiently small. Fixing $p$ and $a$, take a sequence of $z^{\prime}$ s such that $r \rightarrow 0, q \rightarrow p$ and $\epsilon \rightarrow 0$. Noting that $P^{\prime}(0, z) \geq 0$ throughout this sequence, we can pass to the limit in (22) to conclude that

$$
(1+\beta)-2 \geq 0
$$

which, given that $\beta \leq 1$, proves that $\beta=1$. 
To establish the converse, put $\beta=1$ and consider (22) again. We see that for any configuration with $q>r$,

$$
\begin{aligned}
\frac{1}{4} \epsilon^{-2} P^{\prime}(0, z) & =2 \epsilon\left[q^{2}-r^{2}\right]+2 a p[q-r]-2 a\left[q^{2}-r^{2}\right] \\
& >2 a p[q-r]-2 a(q-r)(q+r) \\
& =2 a p[q-r]-2 a p[q-r]=0,
\end{aligned}
$$

where the penultimate equality uses the restriction that $p=q+r$.

Proof of Proposition 4. The central argument behind the proposition is

LEMMA 1. Under the pure contest and large population assumptions, the value of $\hat{\rho}$ must be maximized at a configuration which involves equal population in all groups with nonzero population.

Proof. Use (17), along with the pure contest and large population assumptions, to see that

$$
\begin{aligned}
\frac{1}{\alpha} \hat{\rho} c^{\prime}(\hat{\rho}) & =\sum_{i=1}^{m} \sum_{j \neq i} n_{i}^{2} n_{j}\left[\lambda+\frac{1-\lambda}{n_{i}}\right] \\
& =(2 \lambda-1) \sum_{i=1}^{m} n_{i}^{2}-\lambda \sum_{i=1}^{m} n_{i}^{3}+(1-\lambda) .
\end{aligned}
$$

Pick any population distribution with the property that population is unequal across at least two groups with positive population shares. Without loss of generality $n_{1}=a$ and $n_{2}=b$, and $a>b>0$. Suppose, contrary to our assertion, that such a distribution maximizes $\hat{\rho}$.

For any $\epsilon \in(-b, a-b)$, define $n_{1}(\epsilon) \equiv a-\epsilon, n_{2}(\epsilon) \equiv b+\epsilon$, and $n_{i}(\epsilon) \equiv n_{i}$ for all $i \neq 1,2$, and let

$$
A(\epsilon) \equiv(2 \lambda-1) \sum_{i=1}^{m} n_{i}^{2}(\epsilon)-\lambda \sum_{i=1}^{m} n_{i}^{3}(\epsilon)+(1-\lambda) .
$$

Notice that $A(\epsilon)$ is just the value of $\hat{\rho} c^{\prime}(\hat{\rho}) / \alpha$ when the population distribution is $(a-\epsilon, b+$ $\left.\epsilon, n_{3}, \ldots, n_{m}\right)$. Because $\hat{\rho}$ is maximized at $\mathbf{n}$ by assumption, it must be that $A^{\prime}(0)=0$. Differentiate $A(\epsilon)$ to see that

$$
\begin{aligned}
A^{\prime}(\epsilon) & =-2(2 \lambda-1)\left[n_{1}(\epsilon)-n_{2}(\epsilon)\right]+3 \lambda\left[n_{1}^{2}(\epsilon)-n_{2}^{2}(\epsilon)\right] \\
& =\left[n_{1}(\epsilon)-n_{2}(\epsilon)\right]\left\{3 \lambda\left[n_{1}(\epsilon)+n_{2}(\epsilon)\right]-[4 \lambda-2]\right\}
\end{aligned}
$$

Evaluating the result at $\epsilon=0$, we must conclude that

$$
a+b=\frac{4 \lambda-2}{3 \lambda} \equiv \tau .
$$


Notice in passing that equation (24) already establishes a contradiction when $\lambda \leq 1 / 2$, so from now we presume that $\lambda>1 / 2$.

It is obvious that $\tau \leq 2 / 3$, so there exists $i \neq 1,2$ such that $n_{i}>0$. For every such $i, n_{i}$ must equal either $a$ or $b$. For if this were false for some $i$, we would violate (24) simply by choosing groups 1 and $k$ and redoing the above exercise.

We now claim that for every $i \neq 1,2$ with $n_{i}>0$, we have $n_{i}=a$. Suppose not; then $n_{i}=b$ for some index $i=k$. Permute the labels 1 and $k$, and redo exactly the above exercise with the two groups of equal size $b$. Differentiate again in (23) to see that

$$
A^{\prime \prime}(\epsilon)=4(2 \lambda-1)-6 \lambda\left[n_{1}(\epsilon)+n_{2}(\epsilon)\right]
$$

Evaluate this result at $\epsilon=0$, and use (24) along with $a>b$ to conclude that $A^{\prime \prime}(0)>0$. But this is a contradiction to the maximality of $\mathbf{n}$ : a small population transfer of $\epsilon$ between the two groups of size $b$ will necessarily raise $\hat{\rho}$. This proves the claim.

We must therefore conclude that $\mathbf{n}$ has $S-1$ groups of size $a$ and exactly one group of size $b$, where $a>b$ and $S \geq 3$, and all other groups have zero size. Moreover, (24) holds.

For any $\epsilon \in(-b,[S-1][a-b])$, define $n_{i}(\epsilon) \equiv a-(\epsilon /[S-1])$ for all $i$ such that $n_{i}=a$ and $n_{j}(\epsilon) \equiv b+\epsilon$ for the single group $j$ of size $b$. Define a new function

$$
B(\epsilon) \equiv(2 \lambda-1) \sum_{i=1}^{m} n_{i}^{2}(\epsilon)-\lambda \sum_{i=1}^{m} n_{i}^{3}(\epsilon)+(1-\lambda) .
$$

Note - as in the case of $A(\epsilon)$ - that $B(\epsilon)$ is the value of $\hat{\rho} c^{\prime}(\hat{\rho}) / \alpha$ when the population distribution is $(b+\epsilon ; a-(\epsilon /[S-1]), \ldots, a-(\epsilon /[S-1]))$. Differentiate once to see that

$$
B^{\prime}(\epsilon)=2(2 \lambda-1)\left[b-a+\frac{\epsilon S}{S-1}\right]+3 \lambda\left[\left(a-\frac{\epsilon}{S-1}\right)^{2}-(b+\epsilon)^{2}\right],
$$

and differentiate again to obtain

$$
B^{\prime \prime}(\epsilon)=2(2 \lambda-1) \frac{S}{S-1}-6 \lambda\left[\frac{1}{S-1}\left(a-\frac{\epsilon}{S-1}\right)+(b+\epsilon)\right] .
$$

Evaluate this expression at $\epsilon=0$ to see that

$$
(S-1) B^{\prime \prime}(0)=2(2 \lambda-1) S-6 \lambda[a+b(S-1)] .
$$

Now recall (24) and the fact that $a(S-1)+b=1$ to conclude that

$$
a=\frac{1-\tau}{S-2} \quad \text { and } \quad b=\frac{(S-1) \tau-1}{S-2} .
$$


Using this information in (25), we see that

$$
\begin{aligned}
(S-1) B^{\prime \prime}(0) & =2(2 \lambda-1) S-6 \lambda\left[\frac{1-\tau}{S-2}+\frac{(S-1)^{2} \tau-(S-1)}{S-2}\right] \\
& =2(2 \lambda-1) S-6 \lambda(S \tau-1) \\
& =2[S-\lambda(2 S-3)],
\end{aligned}
$$

where the last equality uses the definition of $\tau$ in (24).

Now $a>b$. Using this information in (26), we see that

$$
1-\tau>(S-1) \tau-1
$$

or equivalently,

$$
S-\lambda(2 S-3)>0 .
$$

Using this information in (27), we must conclude that $B^{\prime \prime}(0)>0$. But now we contradict the presumption that $\mathbf{n}$ maximizes $\hat{\rho}$ : a suitably chosen population transfer between the groups will raise the value of $\hat{\rho}$.

Now we return to the main proof. We observe that when $\hat{\rho}$ is at its maximal value $\hat{U}$, there is only one possible value of $\rho$, and it is $\hat{U}$ as well. To see this, use Lemma 1 to argue that the necessary and sufficient condition for all behavioral correction factors to equal unity (see Proposition 3 and the second example following it) is indeed satisfied. Therefore $\rho$ must equal $\hat{\rho}$.

Define $U(\hat{\rho})$ and $u(\hat{\rho})$ as the maximum and minimum respectively of all possible values of conflict $\rho$, as we range over all population distributions that generate $\hat{\rho}$. By a simple continuity argument, both $U(\hat{\rho})$ and $u(\hat{\rho})$ must converge to $\hat{U}$ as $\hat{\rho} \rightarrow \hat{U}$, which completes the proof. 